\title{
The Lymphocyte Transformation Test Is Useful in the Diagnosis of Fixed Drug Eruption Induced by Etoricoxib
}

Movsisyan $\mathrm{M}^{1}$, Fiandor $\mathrm{A}^{2,3}$, González-Muñoz $\mathrm{M}^{4}$, Quirce $\mathrm{S}^{2,3}$, Bellón $\mathrm{T}^{3,5}$, Hakobyan $\mathrm{A}^{1}$, Marques-Mejias $\mathrm{MA}^{2}$, DomínguezOrtega $\mathrm{J}^{2,3}$, Cabañas $\mathrm{R}^{2,3}$

${ }^{1}$ Department of Allergy, Yerevan State Medical University after Mkhitar Heratsi, Yerevan, Armenia

${ }^{2}$ Allergy Unit, Hospital La Paz, Madrid, Spain.

${ }^{3}$ Hospital La Paz Institute for Health Research (IdiPAZ), Madrid, Spain

${ }^{4}$ Department of Immunology, Hospital La Paz, Madrid, Spain

${ }^{5}$ Resarch Unit. Hospital La Paz Institute for Health Research (IdiPAZ), Madrid, Spain

J Investig Allergol Clin Immunol 2019; Vol. 29(4): 307-309 doi: $10.18176 /$ jiaci.0384

Key words: Fixed drug eruption. Etoricoxib. LTT. Lymphocyte transformation test. Sulfamethoxazole.

Palabras clave: Erupción Fija Medicamentosa. Etoricoxib. TTL. Test de Transformación Linfocitaria. Sulfametoxazol.

Fixed drug eruption (FDE) is a type of cutaneous drug reaction that typically appears as round erythematous and edematous plaques (sometimes with a central blister) at the same site on the skin or mucous membranes and begins 1-2 weeks after the initial exposure to the drug. With subsequent exposures, lesions reactivate within 24 hours and then fade away over several days, often leaving residual, postinflammatory, grayish-brown pigmentation [1].

Antibacterial agents (eg, trimethoprim-sulfamethoxazole, tetracyclines, penicillins) and nonsteroidal anti-inflammatory drugs (NSAIDs) are considered to be the main causes of FDE [1].

Etoricoxib is a selective COX-2 inhibitor. It is usually safe and well tolerated in patients with hypersensitivity to NSAIDs [2]. Nevertheless, some cases of FDE with this drug have been reported $[1,3,4]$, as has a case of symmetrical drugrelated intertriginous and flexural exanthema (SDRIFE) [5] and, recently, a case of anaphylaxis to etoricoxib [6].

The lymphocyte transformation test (LTT) is a useful diagnostic technique in nonimmediate drug reactions [4], although its usefulness in the diagnosis of FDE is open to debate $[7,8]$.

In July 2017, a 46-year-old woman was seen in the allergy unit for an adverse reaction to etoricoxib. She had been diagnosed with cold urticaria in 2007, although this was well controlled with antihistamines. She had experienced 3 episodes of bilateral palpebral angioedema 90 minutes after taking ibuprofen $600 \mathrm{mg}$ in 2008. The diagnostic work-up indicated possible hypersensitivity to NSAIDs. The drug-controlled exposure test performed at that time revealed tolerance to meloxicam $15 \mathrm{mg}$ and etoricoxib $60 \mathrm{mg}$. 


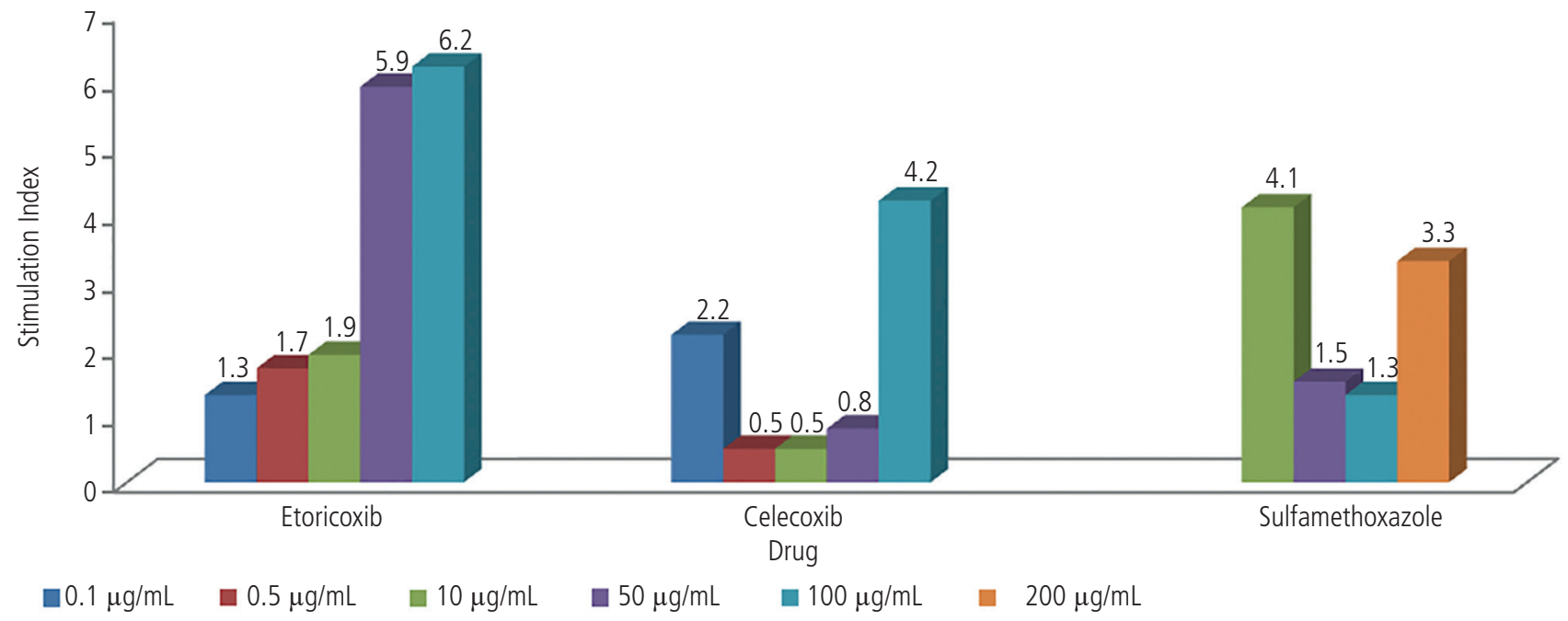

Figure. Results of the Lymphocyte Transformation Test.

In 2016, the patient had received etoricoxib (1 tablet, 5 days) for trochanteritis. She tolerated the therapy well.

In March 2017, the patient noticed pruritic and maculopapular lesions on her face and a single burning macular lesion $(2 \mathrm{~cm})$ twice at the same site on the left side of the abdomen after taking 1 etoricoxib tablet. She was treated with oral antihistamines and topical corticosteroids. The facial lesions disappeared 3 days later. Residual hyperpigmentation was observed on the abdominal lesion. The LTT was performed 4 months after remission according to Pichler and Tilch [7] with etoricoxib, celecoxib, and sulfamethoxazole and was positive with all 3 drugs (Figure). Briefly, peripheral blood mononuclear cells $\left(2 \times 10^{5}\right.$ cells in $200 \mu \mathrm{L}$ ) were stimulated with increasing concentrations of the suspect drugs in triplicate for 6 days in RPMI culture medium supplemented with $5 \%$ autologous serum, and $0.5 \mu \mathrm{Ci}$ of $3 \mathrm{H}$-thymidine was added to the cultures for the last 18 hours. The cell cultures were harvested using glass fiber filters, and radioactivity was measured using a scintillation beta counter. Proliferation was evaluated by incorporating $3 \mathrm{H}$-thymidine to DNA. A stimulation index (SI) was calculated as the ratio of $3 \mathrm{H}$ incorporated by drug-stimulated cultures to baseline incorporation of $3 \mathrm{H}$ by unstimulated cells. The LTT result was considered positive if the SI was higher than 2 [7]. The SI was 5.9 and 6.2 for etoricoxib at $50 \mu \mathrm{g} / \mathrm{mL}$ and $100 \mu \mathrm{g} / \mathrm{mL}$ and 2.2 and 4.2 for celecoxib at $10 \mu \mathrm{g} / \mathrm{mL}$ and $100 \mu \mathrm{g} / \mathrm{mL}$. The SI value for sulfamethoxazole was 4.1 and 3.3 at 10 and $200 \mu \mathrm{g} / \mathrm{mL}$, respectively.

The number of new drugs in the pharmaceutical industry is growing quickly, as is the number of adverse cutaneous drug reactions to these drugs. Approximately 100 drugs have been found to cause FDE, although only 8 cases of etoricoxib-related FDE have been reported $[1,3,4]$.

Etiologic confirmation of FDE is sometimes problematic, particularly in polymedicated patients [1]. Few authors have assessed the diagnostic value of LTT for FDE [7,8]. Pichler and Tilch [7] suggested that LTT in patients with FDE is consistently negative. Nevertheless, Kim et al [8] used LTT to confirm that allopurinol was the culprit drug in a case of allopurinol-induced FDE. We used LTT to confirm etoricoxib as the culprit drug in a patient with FDE and to show possible cross-reactivity with sulfamethoxazole and celecoxib.

Sulfonamides are among the main causes of FDE. Opinions on cross-reactivity between sulfonamide antibiotics and nonantibiotics vary considerably [9]. Muntingh [9] found that patients allergic to sulfonamide antibiotics were more likely to react to sulfonamide nonantibiotics than nonallergic patients. In a recent case report published by our group, the LTT did not reveal cross-reactivity between sulfamethoxazole and sulfadiazine [10]. The new COX-2 inhibitors, such as celecoxib and etoricoxib, contain a sulfone-group. We recorded a positive LTT result with etoricoxib, celecoxib, and sulfamethoxazole. In the case of etoricoxib-induced SDRIFE, the results of patch testing with etoricoxib and celecoxib were positive [5], thus enabling us to hypothesize about possible cross-reactivity between etoricoxib and sulfamethoxazole and also with other drugs containing a sulfone group. However, our in vitro study is limited by the absence of a controlled exposure test and patch tests, with the result that a positive LTT result does not necessarily imply a clinical response.

We think epitopes in sulfonamides may differ between patients and that the antibodies to these drugs could have different reactivities. Therefore, it is necessary to assess crossreactivity on an individual basis.

It is important to emphasize that the patient in the present report took etoricoxib safely for some time before becoming sensitized to the drug. This interesting finding requires further investigation. Etoricoxib may be not as safe as previously thought.

Our results suggest that LTT could be useful for identifying the culprit drug in FDE, a type IV drug hypersensitivity reaction, although this hypothesis should be confirmed in large samples. 


\section{Funding}

The authors declare that no funding was received for the present study.

\section{Conflicts of Interest}

The authors declare that they have no conflicts of interest.

\section{References}

1. Sousa AS, Cardoso JC, Gouveia MP, Gameiro AR, Teixeira VB, Gonçalo M. Fixed drug eruption by etoricoxib confirmed by patch test. An Bras Dermatol. 2016;91(5):652-4.

2. Kowalski ML, Makowska JS, Blanca MS. Hypersensitivity to nonsteroidal anti-inflammatory drugs (NSAIDs) classification, diagnosis and management: review of the EAACI/ENDA and GALEN/HANNA. Allergy. 2011;66:818-29.

3. Andrade $P$, Goncalo M. Fixed drug eruption caused by etoricoxib - 2 cases confirmed by patch testing. Contact Dermatitis. 2011;64:110-20.

4. Ponce $V$, Muñoz-Bellido F, Moreno E, Laffond E, Gonzalez A, Davila I. Fixed drug eruption caused by etoricoxib with tolerance to celecoxib and parecoxib. Contact Dermatitis. 2012;66:106-12.

5. Caralli ME, Seoane Rodríguez M, Rojas Pérez-Ezquerra $P$, Pelta Fernández R, De Barrio Fernández M. Symmetrical Drug-Related Intertriginous and Flexural Exanthema (SDRIFE) Caused by Etoricoxib. J Investig Allergol Clin Immunol. 2016;26(2):128-9.

6. Couto M, López-Salgueiro $R$, Gaspar Â. Anaphylaxis to Etoricoxib. J Investig Allergol Clin Immunol. 2018;28(2):1356.

7. Pichler WJ, Tilch J. The lymphocyte transformation test in the diagnosis of drug hypersensitivity. Allergy. 2004;59:809-20.

8. Kim M-H, Shim E-J, Jung J-W. A Case of AllopurinolInduced Fixed Drug Eruption Confirmed With a Lymphocyte Transformation Test. Allergy Asthma Immunol Res. 2012;4(5):309-10.

9. Muntingh GL. Do some of the newer COX-2 inhibitors crossreact with sulfonamide antibiotics? An overview. S A Orthop J. 2011;10(1):73-6.

10. Monge-Ortega OP, Cabañas R, Fiandor A, Domínguez-Ortega J, González-Muñoz M, Quirce S, et al. Overlap Between DRESS Syndrome and Exanthema Induced by Sulfadiazine in a Patient Treated With Sulfamethoxazole: Utility of the Lymphocyte Transformation Test for Identification of the Culprit Drug. J Investig Allergol Clin Immunol. 2018;28(2):132-4.

Manuscript received October 31, 2018; accepted for publication January 30, 2019.

Rosario Cabañas

Department of Allergy

La Paz University Hospital Health Research Institute (IdiPAZ)

Madrid, Spain

E-mail: mrosario.cabanas@salud.madrid.org 